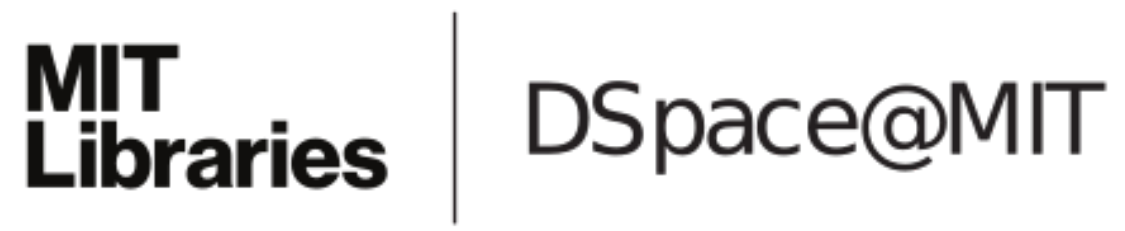

\author{
MIT Open Access Articles
}

Discriminating the progenitor type of supernova remnants with Iron $\mathrm{K}$-shell emission

The MIT Faculty has made this article openly available. Please share how this access benefits you. Your story matters.

Citation: Yamaguchi, Hiroya, Carles Badenes, Robert Petre, Toshio Nakano, Daniel Castro, Teruaki Enoto, Junko S. Hiraga, et al. “Discriminating the Progenitor Type of Supernova Remnants with Iron K-Shell Emission.” The Astrophysical Journal 785, no. 2 (April 3, 2014): L27. (C) 2014 The American Astronomical Society

As Published: http://dx.doi.org/10.1088/2041-8205/785/2//27

Publisher: IOP Publishing

Persistent URL: http://hdl.handle.net/1721.1/92799

Version: Final published version: final published article, as it appeared in a journal, conference proceedings, or other formally published context

Terms of Use: Article is made available in accordance with the publisher's policy and may be subject to US copyright law. Please refer to the publisher's site for terms of use. 


\title{
DISCRIMINATING THE PROGENITOR TYPE OF SUPERNOVA REMNANTS WITH IRON K-SHELL EMISSION
}

\author{
Hiroya Yamaguchi ${ }^{1,2,3}$, Carles Badenes $^{4}$, Robert Petre ${ }^{1}$, Toshio Nakano ${ }^{5}$, Daniel Castro ${ }^{6}$, Teruaki Enoto ${ }^{1,7}$, \\ Junko S. Hiraga ${ }^{5}$, John P. Hughes ${ }^{8}$, Yoshitomo Maeda ${ }^{9}$, Masayoshi Nobukawa ${ }^{10}$, Samar SAFi-Harb ${ }^{11,12}$, \\ Patrick O. Slane ${ }^{3}$, Randall K. Smith ${ }^{3}$, And Hiroyuki Uchida ${ }^{10}$ \\ ${ }^{1}$ NASA Goddard Space Flight Center, Code 662, Greenbelt, MD 20771, USA; hiroya.yamaguchi@ nasa.gov \\ ${ }^{2}$ Department of Astronomy, University of Maryland, College Park, MD 20742, USA \\ ${ }^{3}$ Harvard-Smithsonian Center for Astrophysics, 60 Garden Street, Cambridge, MA 02138, USA \\ ${ }^{4}$ Department of Physics and Astronomy and Pittsburgh Particle Physics, Astrophysics and Cosmology Center (PITT PACC), \\ University of Pittsburgh, 3941 O'Hara Street, Pittsburgh, PA 15260, USA \\ ${ }^{5}$ Department of Physics, The University of Tokyo, Bunkyo, Tokyo 113-0033, Japan \\ ${ }^{6}$ MIT-Kavli Center for Astrophysics and Space Research, 77 Massachusetts Avenue, Cambridge, MA 02139, USA \\ ${ }^{7}$ RIKEN (The Institute of Physical and Chemical Research), 2-1 Hirosawa, Wako, Saitama 351-0198, Japan \\ ${ }^{8}$ Department of Physics and Astronomy, Rutgers University, 136 Frelinghuysen Road, Piscataway, NJ 08854, USA \\ ${ }^{9}$ Institute of Space and Astronautical Science, JAXA, 3-1-1 Yoshinodai, Sagamihara, Kanagawa 229-8510, Japan \\ ${ }^{10}$ Department of Physics, Kyoto University, Kitashirakawa-oiwake-cho, Sakyo-ku, Kyoto 606-8502, Japan \\ ${ }^{11}$ Department of Physics and Astronomy, University of Manitoba, Winnipeg, MB R3T 2N2, Canada \\ Received 2014 March 10; accepted 2014 March 19; published 2014 April 2
}

\begin{abstract}
Supernova remnants (SNRs) retain crucial information about both their parent explosion and circumstellar material left behind by their progenitor. However, the complexity of the interaction between supernova ejecta and ambient medium often blurs this information, and it is not uncommon for the basic progenitor type (Ia or core-collapse) of well-studied remnants to remain uncertain. Here we present a powerful new observational diagnostic to discriminate between progenitor types and constrain the ambient medium density of SNRs using solely Fe K-shell X-ray emission. We analyze all extant Suzaku observations of SNRs and detect $\mathrm{Fe} \mathrm{K} \alpha$ emission from 23 young or middle-aged remnants, including five first detections (IC 443, G292.0+1.8, G337.2-0.7, N49, and N63A). The Fe K $\alpha$ centroids clearly separate progenitor types, with the Fe-rich ejecta in Type Ia remnants being significantly less ionized than in core-collapse SNRs. Within each progenitor group, the $\mathrm{Fe} \mathrm{K} \alpha$ luminosity and centroid are well correlated, with more luminous objects having more highly ionized Fe. Our results indicate that there is a strong connection between explosion type and ambient medium density, and suggest that Type Ia supernova progenitors do not substantially modify their surroundings at radii of up to several parsecs. We also detect a K-shell radiative recombination continuum of Fe in W49B and IC 443, implying a strong circumstellar interaction in the early evolutionary phases of these core-collapse remnants.
\end{abstract}

Key words: ISM: abundances - ISM: supernova remnants - X-rays: ISM

Online-only material: color figure

\section{INTRODUCTION}

Supernova remnants (SNRs) provide unique insights into both the supernova ( $\mathrm{SN}$ ) explosion that generated them and the ambient medium that surrounded their progenitors at the time of the explosion. Unfortunately, the complex physical processes involved in the interaction between ejecta and ambient medium often blur this information, to the point that the explosion type (i.e., Type Ia or core-collapse: Ia and CC hereafter) of several well-studied SNRs still remains controversial.

The X-ray emission from young and middle-aged SNRs is ideally suited to disentangle the contributions from the SN explosion and circumstellar interaction (see Vink 2012 for a recent review). Their thermal X-ray spectra are often dominated by strong optically thin emission lines from ejecta that retain the nucleosynthetic signature of their birth events. On the other hand, the X-ray-emitting plasma is in a state of non-equilibrium ionization (NEI), and its time-dependent ionization degree is controlled by the ambient medium density, which is a sensitive diagnostic of the presence of circumstellar material (CSM) left behind by the SN progenitor (e.g., Badenes et al. 2005, 2007).

\footnotetext{
${ }^{12}$ Canada Research Chair
}

Indeed, much progress has been made in the typing of SNRs using their X-ray emission. Using ASCA data, Hughes et al. (1995) showed that it is possible to distinguish Ia remnants from CC ones by virtue of their ejecta composition; Fe-rich and O-poor SNRs are likely Ia, while SNRs dominated by $\mathrm{O}$ and $\mathrm{Ne}$ lines with weak $\mathrm{Fe} \mathrm{L} \mathrm{emission} \mathrm{are} \mathrm{likely} \mathrm{CC.}$ More recently, Lopez et al. $(2009,2011)$ argued that Chandra images of Ia SNRs show a higher degree of symmetry than those of CC SNRs. This result implies that CC SNe are more asymmetric than Ia SNe, and/or CC SNRs expand into more asymmetric CSM. These methods are promising, but require sophisticated analysis techniques whose results might lead to ambiguous interpretations. Abundance determination in NEI plasmas is notoriously uncertain (see Borkowski et al. 2001, for a discussion), and neither of these methods easily leads to placement of quantitative constraints on the presence of CSM in a SNR. In this Letter, we present a new, straightforward observational diagnostic for typing SNRs in X-rays that relies only on the centroid and flux of a single spectral line-the $\mathrm{Fe}$ $\mathrm{K} \alpha$ emission at $6.4-6.7 \mathrm{keV}$.

The Fe $\mathrm{K}$ line blend is well separated from emission lines of other abundant elements. Since the production of Fe occurs at the heart of an SN explosion, reverse shock heating of this element can be delayed compared to elements synthesized 
in the outer layers. This often results in an ionization state lower than He-like $\left(\mathrm{Fe}^{24+}\right)$ in young or middle-aged SNRs. The ionization state in turn determines the $\mathrm{Fe} \mathrm{K} \alpha$ centroid (e.g., Yamaguchi et al. 2014), which is easily measured using current $\mathrm{CCD}$ instruments. Furthermore, the Fe $\mathrm{K}$ emission is largely unaffected by foreground extinction, unlike $\mathrm{Fe}$ L-shell blends. These spectral advantages and simplicities make our method more straightforward than the existing ones, and especially attractive for current and future X-ray missions with high throughput, like Suzaku, XMM-Newton, and Astro-H. Here we show that the $\mathrm{Fe} \mathrm{K} \alpha$ centroids (hence the Fe ionization state) clearly discriminate the progenitor type and place strong limits on the presence of CSM in SNRs at radii of several parsecs, which has important consequences for SN progenitor studies.

\section{DATA ANALYSIS}

We analyzed archival data of all SNRs that Suzaku has observed to date with the X-ray Imaging Spectrometer (XIS), with no bias nor specific selection criterion. To search for Fe $\mathrm{K} \alpha$ emission, we extracted XIS spectra from the entire X-rayemitting region of each SNR. The only exception was IC 443, which because of its large angular size was only partially imaged by the XIS. For this SNR, we extracted the spectrum from a $10^{\prime}$ diameter circular region in the brightest northern part, and estimated the $\mathrm{Fe} \mathrm{K} \alpha$ flux from the whole SNR by scaling the surface brightness using archival XMM-Newton data. Background subtraction was performed in the following manner: (1) if nearby blank sky data taken with an identical detector operating mode were available, we used them to extract a background spectrum from the same detector region as the source. (2) If the SNR angular size is small enough $\left(d \lesssim 10^{\prime}\right)$ compared with the XIS field of view, background data were taken from the surrounding region. (3) Otherwise, we subtracted only the instrumental, non-X-ray background component (NXB; simulated by the xisnxbgen task), and included models for the extragalactic cosmic X-ray background (aka, $\mathrm{CXB}$ ) and Galactic ridge X-ray emission (GRXE) in our analysis. To estimate the GRXE flux, we followed the relationship between surface brightness and Galactic coordinates described by Uchiyama et al. (2013). As a consistency check, we applied method (3) to all the SNRs which satisfied the criteria for methods (1) and (2), and found no significant change in the measured $\mathrm{Fe} \mathrm{K} \alpha$ blend parameters.

We detected $\mathrm{Fe} \mathrm{K} \alpha$ emission from the 23 SNRs listed in Table 1, including five first detections: G337.2-0.7, IC 443, G292.0+1.8, N49, and N63A. SNRs without detectable Fe K $\alpha$ emission can be categorized into two groups: evolved SNRs whose electron temperature is too low $(\lesssim 1 \mathrm{keV})$ to excite $\mathrm{K}$-shell transitions in $\mathrm{Fe}$ atoms, and young SNRs where the hard X-ray spectrum is dominated by a strong nonthermal continuum. The former category includes most interstellar mediumdominated SNRs (e.g., Cygnus Loop, G156.2+5.7, DEM L71), while the latter includes both shell-like SNRs with cosmic-rayaccelerating blast waves (e.g., G1.9+0.3, RX J1713.7-3946) and plerionic SNRs (e.g., Crab, G21.5-0.9). Recent Chandra observations of G1.9+0.3 separated spatially the thermal emission from the nonthermal continuum, and enabled the detection of Fe K $\alpha$ emission (Borkowski et al. 2010, 2013). Although Suzaku observed this SNR for $\sim 100 \mathrm{ks}$, the Fe K $\alpha$ emission was not spatially resolved and remained undetected. A few SNRs located near the Galactic plane (e.g., G272.2-3.2, Kes 27) showed hints of $\mathrm{Fe} \mathrm{K} \alpha$ emission in their NXB-subtracted spectra, but the fluxes were not significantly larger than those predicted for the GRXE background, so we excluded them from our study.

We fitted the 5-10 keV spectrum of each SNR with a powerlaw (or bremsstrahlung) continuum plus a Gaussian for $\mathrm{Fe} \mathrm{K} \alpha$ emission. Foreground absorption columns are given in Table 1. Some SNRs show emission from $\mathrm{Cr}, \mathrm{Mn}$, and/or $\mathrm{Ni}$, and higher transition series of Fe $\mathrm{K}$ emission, which were modeled using additional Gaussians. A radiative recombination continuum (RRC) of Fe XXV was detected in W49B and IC 443. We modeled this component with an exponential function with a threshold energy of $8.8 \mathrm{keV}$ (corresponding to the ionization potential of $\mathrm{Fe}^{24+}$, following Ozawa et al. 2009). This is the first detection of the Fe RRC from IC 443, indicating that Fe atoms in this remnant are significantly overionized, similar to Si and S (Yamaguchi et al. 2009). The result is presented in more detail in a separate paper (Ohnishi et al. 2014). We list the measured centroid energy and unabsorbed flux of the $\mathrm{Fe} \mathrm{K} \alpha$ blend for each SNR in Table 1.

\section{DISCUSSION}

Figure 1 shows the $\mathrm{Fe} \mathrm{K} \alpha$ centroid energy and line luminosity for each SNR, together with the corresponding effective charge state $\left\langle z_{\mathrm{Fe}}\right\rangle$ (Yamaguchi et al. 2014). The line luminosities were calculated from the derived unabsorbed fluxes using the distances given in Table 1. The uncertainty in distance to the Galactic SNRs is assumed to be $\pm 10 \%$ of the mean values. Although some sources have larger uncertainties (e.g., G349.7+0.2, see Tian \& Leahy 2014), the fundamental result is not affected, since the line centroids play a more important role than the luminosities in typing SNRs as described below. Also shown in Figure 1 are theoretical predictions for SNR models derived from Chandrasekhar-mass Type Ia SN ejecta profiles evolving in a uniform ambient medium density in the range of (1-5) $\times 10^{-24} \mathrm{~g} \mathrm{~cm}^{-3}$ for SNR ages of up to $5000 \mathrm{yr}$ (see Badenes et al. 2003, 2005, 2006, for details on the models). The ejecta profiles shown include three delayed detonation explosion models spanning the nominal range of SN Ia kinetic energies and ${ }^{56} \mathrm{Ni}$ yields (DDTa, DDTc, and DDTg), and two pulsating delayed detonation explosions (PDDa and PDDc). The predicted $\mathrm{Fe} \mathrm{K} \alpha$ centroids and luminosities are calculated using updated atomic data (Yamaguchi et al. 2014). The efficiency of collisionless electron heating at the reverse shock is set so that the electron-to-ion temperature ratio is $0.01-0.03$ at the immediate post-shock region (Badenes et al. 2005; Yamaguchi et al. 2014). Similar models for CC SNRs are not available in the literature, but the Ia SNR models and the distribution of the data points in Figure 1 allow us to make a number of important interpretations using our SNR sample.

First, all bona fide Ia SNRs have an $\mathrm{Fe} \mathrm{K} \alpha$ centroid of $\lesssim 6550 \mathrm{keV}\left(\left\langle z_{\mathrm{Fe}}\right\rangle \lesssim 20\right)$, while all bona fide CC SNRs have a higher centroid energy. The separation is very clear: no single object with a robust progenitor type (i.e., from a known association to a compact object, or light echo spectroscopy, or detailed modeling of the ejecta emission) falls on the wrong side of the centroid boundary. Since the Ia and CC SNRs in our sample have similar ages and radii, this large difference in the ionization state must be due to significantly higher ambient medium densities in the CC SNRs. This is in line with the expectations from stellar evolution models, which predict significant (several $M_{\odot}$ ) mass loss from CC SN progenitors, either due to winds or binary evolution (Langer 2012). In any case, the clear division in $\mathrm{Fe} \mathrm{K} \alpha$ centroid allows us to firmly establish the classification of several objects with unclear or 
Table 1

List of the SNRs Where Fe K $\alpha$ Emission is Detected ${ }^{\mathrm{a}}$

\begin{tabular}{|c|c|c|c|c|c|c|c|c|c|c|}
\hline Name $^{b}$ & Obs. ID & $\begin{array}{l}\text { Exposure } \\
(\mathrm{ks})\end{array}$ & $\begin{array}{l}\text { Energy } \\
(\mathrm{eV})\end{array}$ & $\begin{array}{l}\text { Photon Flux } \\
\left(10^{-5} \mathrm{~cm}^{-2} \mathrm{~s}^{-1}\right)\end{array}$ & $\begin{array}{c}N_{\mathrm{H}^{\mathrm{c}}} \\
\left(10^{22} \mathrm{~cm}^{-2}\right)\end{array}$ & $\begin{array}{l}\text { Distance } \\
(\mathrm{kpc})\end{array}$ & $\begin{array}{l}\text { Radius } \\
\text { (pc) }\end{array}$ & $\begin{array}{l}\text { Age } \\
(\mathrm{yr})\end{array}$ & $\mathrm{BGD}^{\mathrm{d}}$ & Refs. ${ }^{e}$ \\
\hline \multicolumn{11}{|c|}{ Type Ia SNRs and Candidates } \\
\hline Kepler & $5050920[1-7] 0$ & 574 & $6438 \pm 1$ & $34.6 \pm 0.2$ & 0.5 & 4.8 & 2.4 & 410 & (1) & 1 \\
\hline $3 \mathrm{C} 397^{\dagger}$ & 505008010 & 69 & $6556_{-3}^{+4}$ & $13.7 \pm 0.4$ & 3.0 & 10.3 & 10.5 & $1500-5500$ & (2) & 2,3 \\
\hline Tycho* & $5030850[1,2] 0$ & 416 & $6431 \pm 1$ & $61.0 \pm 0.4$ & 0.7 & 2.8 & 3.4 & 442 & (2) & 4,5 \\
\hline $\mathrm{RCW} 86^{\dagger}$ & (See notes) & 378 & $6408_{-5}^{+4}$ & $14.0 \pm 0.7$ & 0.3 & 2.5 & 16 & 1829 & (3) & 6 \\
\hline SN $1006^{*}$ & (See notes) & 317 & $6429 \pm 10$ & $2.55 \pm 0.43$ & 0.07 & 2.2 & 10 & 1008 & (3) & 7 \\
\hline G337.2-0.7 & 507068010 & 304 & $6505_{-31}^{+26}$ & $0.21 \pm 0.06$ & 4.0 & 9.3 & 8.1 & $5000-7000$ & (2) & 8 \\
\hline G344.7-0.1 ${ }^{\dagger}$ & 501011010 & 42 & $6463_{-10}^{+9}$ & $4.03 \pm 0.33$ & 5.0 & 14 & 16 & $3000-6000$ & (2) & 9 \\
\hline G352.7-0.1 $1^{\dagger}$ & 506052010 & 202 & $6443_{-12}^{+8}$ & $0.82 \pm 0.08$ & 2.6 & 7.5 & 6.0 & $\sim 5000$ & (2) & 10 \\
\hline $\mathrm{N} 103 \mathrm{~B}^{\dagger}$ & 804039010 & 224 & $6545 \pm 6$ & $2.15 \pm 0.10$ & 0.06 & 50 & 3.6 & $\sim 860$ & (2) & 11,12 \\
\hline $0509-67.5^{*}$ & $5080720[1,2] 0$ & 329 & $6425_{-15}^{+14}$ & $0.32 \pm 0.04$ & 0.05 & 50 & 3.6 & $\sim 400$ & (2) & 12,13 \\
\hline $0519-69.0^{*}$ & 806026010 & 348 & $6498_{-8}^{+6}$ & $0.93 \pm 0.05$ & 0.06 & 50 & 4.0 & $\sim 600$ & (2) & 12,14 \\
\hline \multicolumn{11}{|c|}{ Core-collapse SNRs and Candidates } \\
\hline Sgr A East* & (See notes) & 88 & $6664 \pm 3$ & $22.3 \pm 1.0$ & 10 & 8.5 & 3.7 & $\sim 4000$ & (2) & 15 \\
\hline $\mathrm{G} 0.61+0.01^{\dagger}$ & 100037060 & 77 & $6634_{-12}^{+14}$ & $3.3 \pm 0.5$ & 16 & 8.5 & 5.0 & $\sim 7000$ & (2) & 16 \\
\hline W49B & $50308[4,5] 010$ & 114 & $6663 \pm 1$ & $109 \pm 1$ & 5.0 & 8.0 & 5.8 & $1000-3000$ & (2) & 17 \\
\hline Cas A* & 100043020 & 7 & $6617_{-2}^{+3}$ & $435 \pm 9$ & 2.0 & 3.4 & 2.7 & $310-350$ & (2) & 18 \\
\hline IC 443 & $5070150[1-4] 0$ & 368 & $6674_{-13}^{+10}$ & $6.01 \pm 0.59$ & 0.6 & 1.5 & 10 & $4000-30000$ & (3) & 19 \\
\hline G292.0+1.8* & 506062010 & 44 & $6585_{-28}^{+27}$ & $1.38 \pm 0.35$ & 0.5 & 6.2 & 11 & $\sim 3000$ & (3) & 20 \\
\hline G349.7+0.2 & 506064010 & 160 & $6617_{-6}^{+7}$ & $2.92 \pm 0.18$ & 7.0 & 11.5 & 4.0 & $\sim 3500$ & (2) & 21,22 \\
\hline G350.1-0.3* & 506065010 & 70 & $6587_{-10}^{+11}$ & $2.24 \pm 0.23$ & 3.7 & 4.5 & 1.3 & $\sim 900$ & (2) & 23 \\
\hline $\mathrm{N} 49^{\dagger}$ & 807007010 & 185 & $6628_{-26}^{+29}$ & $0.18 \pm 0.04$ & 0.06 & 50 & 8.5 & $\sim 6600$ & (2) & 24 \\
\hline N63A & 508071010 & 82 & $6647_{-17}^{+16}$ & $0.86 \pm 0.12$ & 0.06 & 50 & 10 & $2000-5000$ & (2) & 25 \\
\hline N132D & (See notes) & 86 & $6656 \pm 9$ & $1.83 \pm 0.17$ & 0.06 & 50 & 13 & $\sim 3150$ & (2) & 26 \\
\hline SN 1987A* & 707020010 & 81 & $6646_{-54}^{+55}$ & $0.19 \pm 0.08$ & 0.06 & 50 & 0.2 & 27 & (2) & 27 \\
\hline
\end{tabular}

Notes. Observation ID—RCW 86: 503004010, 501037010, 503001010, 503002010, 503003010, 503004010; SN 1006: 500016010, 500017010, 502046010; Sgr A east: 100027010, 100037040, 100048010; N132D: 105011010, 106010010, 106010020.

a The uncertainties are in the $90 \%$ confidence range.

$\mathrm{b}$ The asterisks $(*)$ indicate the SNRs for which classification is robust from a known association with a compact object, light echo spectroscopy, and/or detailed modeling of the ejecta emission. Daggers $(\dagger)$ indicate that the progenitor type of these SNRs is controversial or unknown.

${ }^{c}$ Absorption column density with the solar elemental composition (Wilms et al. 2000). For the LMC SNRs, only the Galactic component (Dickey \& Lockman $1990)$ is shown, but the absorption in the LMC $\left(\lesssim 10^{21} \mathrm{~cm}^{-2}\right)$ does not affect the spectra above $5 \mathrm{keV}$.

${ }^{\mathrm{d}}$ Background subtraction method we applied (see text in Section 2).

' Representative references: (1) Reynolds et al. 2007; (2) Chen et al. 1999; (3) Safi-Harb et al. 2005; (4) Badenes et al. 2006; (5) Tian \& Leahy 2011; (6) Williams et al. 2011; (7) Yamaguchi et al. 2008; (8) Rakowski et al. 2006; (9) Yamaguchi et al. 2012; (10) Giacani et al. 2009; (11) Lewis et al. 2003; (12) Rest et al. 2005; (13) Warren \& Hughes 2004; (14) Kosenko et al. 2010; (15) Koyama et al. 2007b; (16) Koyama et al. 2007a; (17) Keohane et al. 2007; (18) Hwang \& Laming 2012; (19) Troja et al. 2008; (20) Park et al. 2004; (21) Lazendic et al. 2005; (22) Tian \& Leahy 2014; (23) Gaensler et al. 2008; (24) Park et al. 2012; (25) Warren et al. 2003; (26) Borkowski et al. 2007; (27) Maggi et al. 2012.

controversial types. Both RCW 86 and G344.7-0.1 were once considered to be CC SNRs (e.g., Vink et al. 1997; Lopez et al. 2011), but recent observations have suggested their Ia origin (e.g., Williams et al. 2011; Yamaguchi et al. 2012). The latter is supported by our study. The $\mathrm{Fe} \mathrm{K} \alpha$ centroid of the Ia SNR G337.2-0.7 (Rakowski et al. 2006) falls a bit outside the range of our theoretical models, but this is not surprising given the estimated age (5000-7000 yr). Giacani et al. (2009) suggested that the highly absorbed SNR G352.7-0.1 might have been a $\mathrm{CC}$ event, but its $\mathrm{Fe} \mathrm{K} \alpha$ centroid puts it squarely in the Ia region. The Fe K $\alpha$ centroid for G1.9+0.3 reported by Borkowski et al. (2013) also falls within the Ia region, supporting the typing of this SNR by Borkowski et al. (2010). Likewise, several SNRs suspected to be of a CC origin without confirmed associations with compact objects (e.g., G0.61+0.01: Koyama et al. 2007a; N49: Park et al. 2012; N63A: Warren et al. 2003; N132D:
Borkowski et al. 2007) fall clearly in the CC region due to their high centroid energies. From the $\mathrm{Fe} \mathrm{K} \alpha$ centroid alone, the classification for 3C 397 and N103B is somewhat unclear. We emphasize, however, that the observed $\mathrm{Fe} \mathrm{K} \alpha$ parameters of both SNRs can be well reproduced by our Ia SNR models. If 3C 397 is indeed a Type Ia remnant (as suggested by Chen et al. 1999), it should be relatively old ( $\approx 3000 \mathrm{yr}$ ) and has evolved in a high-density interstellar medium $\left(\sim 5 \times 10^{-24} \mathrm{~g} \mathrm{~cm}^{-3}\right)$, given the comparison with our model plots. These values are consistent with the estimates of Safi-Harb et al. (2005), although a CC origin was suggested in their work. The explosion type of N103B has also been a matter of controversy (e.g., van der Heyden et al. 2002; Lewis et al. 2003), but we favor the Ia hypothesis, based on the high maximum luminosity of its parent explosion inferred from the light echo data (Rest et al. 2005), in addition to the properties of the $\mathrm{Fe} \mathrm{K} \alpha$ emission. 

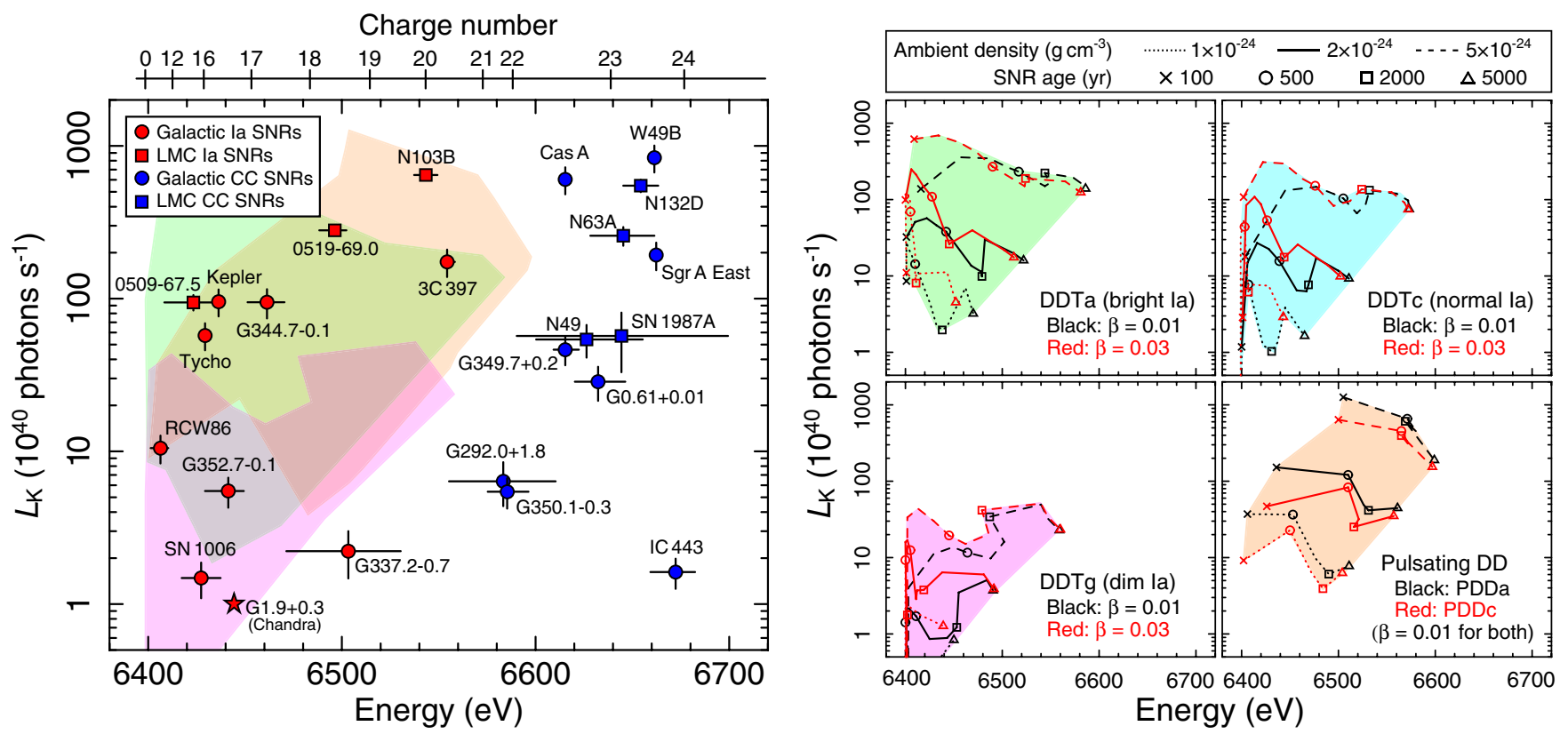

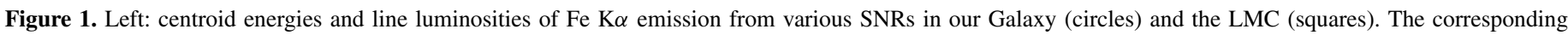

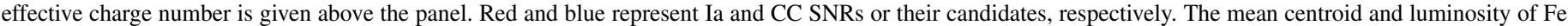

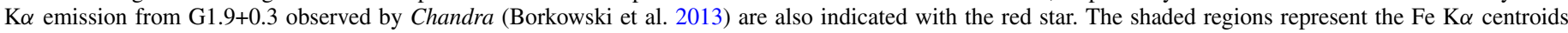

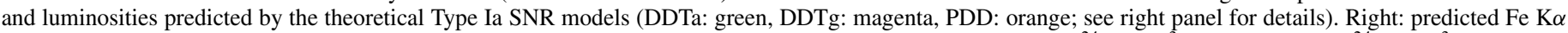

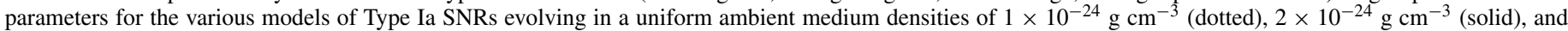
$5 \times 10^{-24} \mathrm{~g} \mathrm{~cm}^{-3}$ (dashed). Crosses, circles, squares, and triangles indicate SNR ages of $100 \mathrm{yr}, 500 \mathrm{yr}, 2000 \mathrm{yr}$, and $5000 \mathrm{yr}$, respectively.

(A color version of this figure is available in the online journal.)

Second, within each group, the centroids and line luminosities are fairly well correlated, such that SNRs with more highly ionized Fe tend to have more luminous $\mathrm{Fe} \mathrm{K} \alpha$ lines. This is likely a consequence of the NEI characteristics of the emitting plasma; in order to collisionally ionize $\mathrm{Fe}$ atoms to higher states, higher post-shock densities are required, which also result in higher emission measures and thus X-ray luminosities. The SNR age also plays a role in the ionization state of the ejecta, but ambient medium density is the main driver, as illustrated by the Ia SNR models in Figure 1. Interestingly, both SNR types span a similar range of $\mathrm{Fe} \mathrm{K} \alpha$ luminosities, despite the fact that the typical ejected mass of $\mathrm{Fe}\left({ }^{56} \mathrm{Ni}\right)$ is an order of magnitude smaller in CC than in Ia SNe (Woosley \& Weaver 1995; Iwamoto et al. 1999). This is again due to the higher emission measure in the CC SNRs associated with higher post-shock density, and is one of the reasons why typing SNRs has been difficult without having a detailed model for their dynamics and plasma evolution. In this context, the relatively high $\mathrm{Fe} \mathrm{K} \alpha$ centroid and luminosity for SN 1987A are particularly interesting, given the young age and low ejected Fe mass of this SNR. The progenitors of the overionized SNRs, W49B and IC 443, might have an especially high mass loss rate, leading to a CSM dense enough to produce a strong circumstellar interaction in the early evolutionary phase of their remnants (e.g., Yamaguchi et al. 2009).

Finally, although our Ia SNR models are relatively simplistic (one-dimensional hydrodynamics in uniform ambient density), the parameter space they span includes all Ia SNRs. This is remarkable in its own right, because some Ia SNRs, like Kepler (Reynolds et al. 2007) and N103B (Lewis et al. 2003), are known to be interacting with a nonuniform ambient medium. Our analysis does not rule out the presence of CSM in these objects, but simply indicates that deviations from a uniform ambient medium in Ia SNRs, if present, cannot be very large, and rules out large CSM masses (several $M_{\odot}$ ) as seen in CC
SNRs. Middle-aged Ia SNRs with low $\mathrm{Fe} \mathrm{K} \alpha$ centroids and luminosities might be interacting with an exceptionally lowdensity interstellar medium (e.g., SN1006: Yamaguchi et al. 2008), or with a low-density wind-blown cavity excavated by the progenitor (e.g., RCW 86: Williams et al. 2011). On the other hand, young Ia SNRs with higher $\mathrm{Fe} \mathrm{K} \alpha$ centroids and luminosities, like N103B (Lewis et al. 2003), might be interacting with some kind of CSM, but their Fe K $\alpha$ emission can also be explained by uniform ambient density models, at least at the level of detail allowed by our study.

\section{CONCLUSIONS}

We have presented a systematic analysis of $\mathrm{Fe} \mathrm{K} \alpha$ emission from 23 Galactic and LMC SNRs observed by Suzaku. We find that the $\mathrm{Fe} \mathrm{K} \alpha$ line luminosities of Type Ia and CC SNRs are distributed in a similar range $\left(L_{\mathrm{K}}=10^{40-43}\right.$ photons s$\left.^{-1}\right)$, but the $\mathrm{Fe} \mathrm{K} \alpha$ centroid energies clearly distinguish Ia from CC SNRs, with the former always having centroids below $\sim 6.55 \mathrm{keV}$ and the latter always above. We interpret this separation as a signature of different mass loss rates in Ia and CC SN progenitors. The $\mathrm{Fe} \mathrm{K} \alpha$ emission of all the Ia objects in our sample is compatible with SNR models that expand into a uniform ambient medium, which suggests that Ia progenitors do not modify their surroundings as strongly as $\mathrm{CC}$ progenitors do. This is in line with known limits from prompt X-ray (Hughes et al. 2007) and radio (Chomiuk et al. 2012) emission from Ia $\mathrm{SNe}$, but our results probe a different regime, constraining the structure of the CSM to larger radii (several parsecs) and progenitor mass loss rates further back in the pre-SN evolution of the progenitor. A quantification of these constraints and a more detailed analysis of the CC SNR sample are left for future work.

The full potential of our method will be realized when it is applied to larger samples of higher quality data, as will be 
accessible to high resolution spectrometers like those on Astro- $H$ and other future missions with large effective areas in the $\mathrm{Fe} \mathrm{K} \alpha$ band like Athena. These instruments will open the possibility of studying statistically significant samples of X-ray-emitting SNRs in nearby galaxies with resolved stellar populations like M31, which will in turn dramatically increase our knowledge of both Type Ia and CC SN progenitors.

We are grateful to Dr. Katsuji Koyama for providing Suzaku data he obtained as a Principal Investigator and kindly reviewing our manuscript. We also thank Drs. Kazimierz J. Borkowski, Thomas M. Dame, Adam, R. Foster, John D. Raymond, Satoru Katsuda, Toshiki Sato, and Hideki Uchiyama for helpful discussion and suggestions in preparing this Letter.

\section{REFERENCES}

Badenes, C., Borkowski, K. J., \& Bravo, E. 2005, ApJ, 624, 198

Badenes, C., Borkowski, K. J., Hughes, J. P., Hwang, U., \& Bravo, E. 2006, ApJ, 645, 1373

Badenes, C., Bravo, E., Borkowski, K. J., \& Domínguez, I. 2003, ApJ, 593, 358

Badenes, C., Hughes, J. P., Bravo, E., \& Langer, N. 2007, ApJ, 662, 472

Borkowski, K. J., Hendrick, S. P., \& Reynolds, S. P. 2007, ApJL, 671, L45

Borkowski, K. J., Lyerly, W. J., \& Reynolds, S. P. 2001, ApJ, 548, 820

Borkowski, K. J., Reynolds, S. P., Green, D. A., et al. 2010, ApJL, 724, L161

Borkowski, K. J., Reynolds, S. P., Hwang, U., et al. 2013, ApJL, 771, L9

Chen, Y., Sun, M., Wang, Z.-R., \& Yin, Q. F. 1999, ApJ, 520, 737

Chomiuk, L., Soderberg, A. M., Moe, M., et al. 2012, ApJ, 750, 164

Dickey, J. M., \& Lockman, F. J. 1990, ARA\&A, 28, 215

Gaensler, B. M., Tanna, A., Slane, P. O., et al. 2008, ApJL, 680, L37

Giacani, E., Smith, M. J. S., Dubner, G., et al. 2009, A\&A, 507, 841

Hughes, J. P., Chugai, N., Chevalier, R., Lundqvist, P., \& Schlegel, E. 2007, ApJ, 670,1260

Hughes, J. P., Hayashi, I., Helfand, D., et al. 1995, ApJL, 444, L81

Hwang, U., \& Laming, J. M. 2012, ApJ, 746, 130
Iwamoto, K., Brachwitz, F., Nomoto, K., et al. 1999, ApJS, 125, 439

Keohane, J. W., Reach, W. T., Rho, J., \& Jarrett, T. H. 2007, ApJ, 654, 938

Kosenko, D., Helder, E. A., \& Vink, J. 2010, A\&A, 519, A11

Koyama, K., Inui, T., Hyodo, Y., et al. 2007a, PASJ, 59, S221

Koyama, K., Uchiyama, H., Hyodo, Y., et al. 2007b, PASJ, 59, S237

Langer, N. 2012, ARA\&A, 50, 107

Lazendic, J. S., Slane, P. O., Hughes, J. P., Chen, Y., \& Dame, T. M. 2005, ApJ, 618,733

Lewis, K. T., Burrows, D. N., Hughes, J. P., et al. 2003, ApJ, 582, 770

Lopez, L. A., Ramirez-Ruiz, E., Badenes, C., et al. 2009, ApJL, 706, L106

Lopez, L. A., Ramirez-Ruiz, E., Huppenkothen, D., Badenes, C., \& Pooley, D. A. 2011, ApJ, 732, 114

Maggi, P., Haberl, F., Sturm, R., \& Dewey, D. 2012, A\&A, 548, L3

Ohnishi, T., Uchida, H., Tsuru, T. G., et al. 2014, ApJ, 784, 74

Ozawa, M., Koyama, K., Yamaguchi, H., Masai, K., \& Tamagawa, T. 2009, ApJL, 706, L71

Park, S., Hughes, J. P., Slane, P. O., et al. 2004, ApJL, 602, L33

Park, S., Hughes, J. P., Slane, P. O., et al. 2012, ApJ, 748, 117

Rakowski, C. E., Badenes, C., Gaensler, B. M., et al. 2006, ApJ, 646, 982

Rest, A., Suntzeff, N. B., Olsen, K., et al. 2005, Natur, 438, 1132

Reynolds, S. P., Borkowski, K. J., Hwang, U., et al. 2007, ApJL, 668, L135

Safi-Harb, S., Dubner, G., Petre, R., Holt, S. S., \& Durouchoux, P. 2005, ApJ, 618,321

Tian, W. W., \& Leahy, D. A. 2011, ApJL, 729, L15

Tian, W. W., \& Leahy, D. A. 2014, ApJL, 783, L2

Troja, E., Bocchino, F., Miceli, M., \& Reale, F. 2008, A\&A, 485, 777

Uchiyama, H., Nobukawa, M., Tsuru, T. G., \& Koyama, K. 2013, PASJ, 65, 19

van der Heyden, K. J., Behar, E., Vink, J., et al. 2002, A\&A, 392, 955

Vink, J. 2012, A\&ARv, 20, 49

Vink, J., Kaastra, J. S., \& Bleeker, J. A. M. 1997, A\&A, 328, 628

Warren, J. S., \& Hughes, J. P. 2004, ApJ, 608, 261

Warren, J. S., Hughes, J. P., \& Slane, P. O. 2003, ApJ, 583, 260

Williams, B. J., Blair, W. P., Blondin, J. M., et al. 2011, ApJ, 741, 96

Wilms, J., Allen, A., \& McCray, R. 2000, ApJ, 542, 914

Woosley, S. E., \& Weaver, T. A. 1995, ApJS, 101, 181

Yamaguchi, H., Eriksen, K. A., Badenes, C., et al. 2014, ApJ, 780, 136

Yamaguchi, H., Koyama, K., Katsuda, S., et al. 2008, PASJ, 60, S141

Yamaguchi, H., Ozawa, M., Koyama, K., et al. 2009, ApJL, 705, L6

Yamaguchi, H., Tanaka, M., Maeda, K., et al. 2012, ApJ, 749, 137 